Int. J. Dev. Biol. 60: 305-314 (2016)

doi: $10.1387 / \mathrm{ijdb} .160134 \mathrm{jk}$

\title{
Flexibility vs. robustness in cell cycle regulation of timing of M-phase entry in Xenopus laevis embryo cell-free extract
}

\author{
MATEUSZ DEBOWSKI 1,\#, MOHAMMED EL DIKA ${ }^{2,3, \#, ~ J A C E K ~ M A L E J C Z Y K ~}{ }^{4}$, ROBERT ZDANOWSKI5, \\ CLAUDE PRIGENT ${ }^{2,3}$, JEAN-PIERRE TASSAN ${ }^{2,3}$, MALGORZATA KLOC ${ }^{6}$, MIROSLAW LACHOWICZ*,1 $^{*}$ \\ and JACEK Z. KUBIAK ${ }^{*, 2,3,5}$
}

\begin{abstract}
${ }^{1}$ Institute of Applied Mathematics and Mechanics, Faculty of Mathematics, Informatics and Mechanics, University of Warsaw, Warsaw, Poland, ${ }^{2}$ CNRS, UMR 6290, Institute of Genetics and Development of Rennes, Cell Cycle Group, Rennes, France, ${ }^{3}$ University Rennes 1, UEB, IFR 140, Faculty of Medicine, Rennes, France, ${ }^{4}$ Medical University of Warsaw, Department of Histology \& Embryology, Warsaw, Poland, ${ }^{5}$ Department of Regenerative Medicine, Military Institute of Hygiene and Epidemiology, Warsaw, Poland and ${ }^{6}$ The Methodist Hospital Research Institute, Bertner Ave, Houston, TX, USA
\end{abstract}

\begin{abstract}
During the cell cycle, cyclin dependent kinase 1 (CDK1) and protein phosphatase 2A (PP2A) play major roles in the regulation of mitosis. CDK1 phosphorylates a series of substrates triggering M-phase entry. Most of these substrates are dephosphorylated by PP2A. To allow phosphorylation of CDK1 substrates, PP2A is progressively inactivated upon M-phase entry. We have shown previously that the interplay between these two activities determines the timing of M-phase entry. Slight diminution of CDK1 activity by the R03306 inhibitor delays M-phase entry in a dose-dependent manner in Xenopus embryo cell-free extract, while reduction of PP2A activity by $\mathrm{OA}$ inhibitor accelerates this process also in a dose-dependent manner. However, when a mixture of R03306 and OA is added to the extract, an intermediate timing of M-phase entry is observed. Here we use a mathematical model to describe and understand this interplay. Simulations showing acceleration and delay in M-phase entry match previously described experimental data. CDC25 phosphatase is a major activator of CDK1 and acts through CDK1 Tyr15 and Thr14 dephosphorylation. Addition of CDC25 activity to our mathematical model was also consistent with our experimental results. To verify whether our assumption that the dynamics of CDC25 activation used in this model are the same in all experimental variants, we analyzed the dynamics of CDC25 phosphorylation, which reflect its activation. We confirm that these dynamics are indeed very similar in control extracts and when RO3306 and OA are present separately. However, when R03306 and OA are added simultaneously to the extract, activation of CDC25 is slightly delayed. Integration of this parameter allowed us to improve our model. Furthermore, the pattern of CDK1 dephosphorylation on Tyr15 showed that the real dynamics of CDK1 activation are very similar in all experimental variants. The model presented here accurately describes, in mathematical terms, how the interplay between CDK1, PP2A and CDC25 controls the flexible timing of M-phase entry.
\end{abstract}

KEY WORDS: cell cycle regulation, $M$-phase entry, CDK, PP2A, CDC25, mathematical model

Introduction

Cell cycle control is driven in large part by phosphorylation and dephosphorylation reactions carried out by kinases and protein phosphatases, respectively (Mochida \& Hunt, 2012). The major enzymes that control M-phase entry are Cyclin-Dependent Kinase 1 (CDK1) and protein phosphatase 2A (PP2A), which phosphorylate
(CDK1) and dephosphorylate (PP2A) specific target substrates (Hunt, 2013). The activities of CDK1 and PP2Aare also regulated by interlaced feedback loops of phosphorylation and dephosphorylation (Fisher et al., 2012). Most proteins phosphorylated by CDK1 upon

Abbreviations used in this paper: CDK, cyclin dependent kinase; PP2A, protein phosphatase 2A.

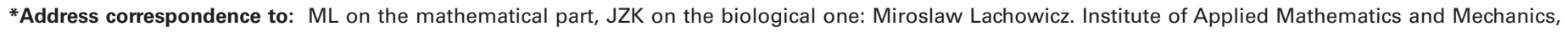

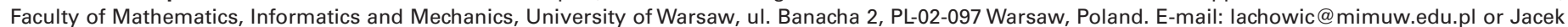

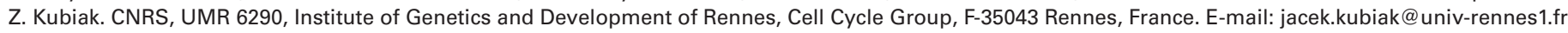

\#Note: These authors equally contributed to this paper: MD to the mathematical part, MED to the biological part.

Accepted: 3 May 2016.

ISSN: Online 1696-3547, Print 0214-6282

(c) 2016 UPV/EHU Press

Printed in Spain 


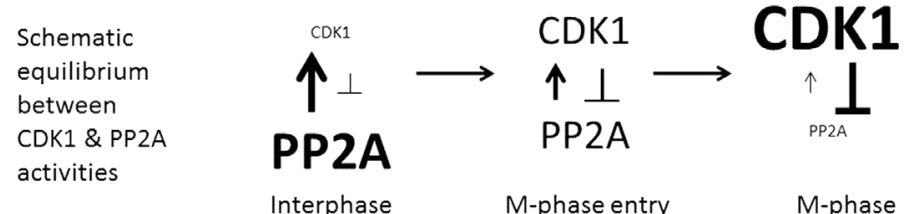

Fig. 1. Equilibrium between CDK1 and PP2A. We show here only the general tendencies between the two antagonistic activities: phosphorylation and dephosphorylation of the same substrate. We do not take into account the complex regulation via Greatwall kinase and PP1.

M-phase entry are dephosphorylated by PP2A. Phosphorylation of CDK1 substrates is a key process for M-phase initiation. To allow accumulation of phosphorylated CDK1 substrates and subsequent onset of mitosis, the PP2A phosphatase is inhibited by a CDK1dependent mechanism involving Greatwall kinase (Lorca and Castro, 2013). Thus, when CDK1 is progressively activated, PP2A becomes progressively repressed (Fig. 1). However, little is known about the dynamics of these processes and how the equilibrium between these two enzymes regulates the timing of M-phase entry.

Cell cycle regulation is extremely robust. For instance, in the strain of fission yeast Schizosaccharomyces pombe, in which cyclin $B$ is fused with $C D K 1$, individual cells contain significantly different levels of cyclin B (Coudreuse \& Nurse, 2010). Despite this, the temporal control of mitosis is respected and all cells enter M-phase while very similar in size, which suggests that the timing of mitosis is similar in cells with different levels of cyclin B (Coudreuse \& Nurse, 2010). This robustness in mitotic timing control shows that cells have mechanisms that accelerate M-phase entry when cyclin B levels are relatively low and delay it when cyclin B levels are high, which allows equilibration of the timing between different cells. This is achieved through the function of a complex regulatory network that controls cell cycle events. For instance, CDK1 requires the activity of the CDC25 phosphatase to be activated (Kumagai \& Dunphy, 1991). CDC25-dependent activation of CDK1 is counterbalanced by Myt1/Wee1 kinases (Fattaey \& Booher, 1997). The process of CDK1 activation is also slowed down, independently of the CDC25/Myt1/Wee1 loop, by a CDC6-dependent inhibitory mechanism inhibiting CDK1 (El Dika et al., 2014a). Finally, the levels of CDK1-phosphorylated substrates, which effectively induce M-phase entry, can be counterbalanced to different extent by the PP2A phosphatase (El Dika et al., 2014b). Any alteration of these elements of the controlling network immediately provokes a compensatory response from another element(s) restoring the

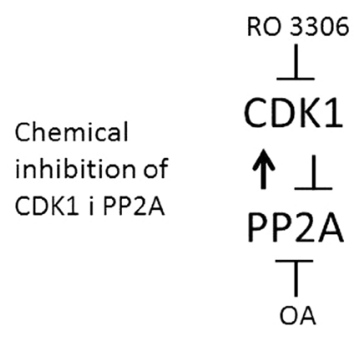

Fig 2 (left). Inhibitory action of OA and R03306 on PP2A and CDK1.

Fig. 3 (right). How OA and R03306 modify the timing of M-phase entry. CDK1 is labeled in red when active. (1) control; (2) RO3306; (3) $O A$; (4) $R 03306+O A$. equilibrium. Therefore, cells with high levels of cyclin B may not activate CDK1 sooner than those with low levels of cyclin $B$ because the initial CDK1 activity may be balanced by higher Myt1/Wee1, CDC6 or PP2A, as well as by another, unknown component of the controlling network.

Similar to the situation described above for S. pombe (Coudreuse \& Nurse, 2010), embryonic cell divisions are generally stereotyped in time (Hartley et al., 1996). However, in Xenopus laevis embryo cell-free extracts, moderate modifications of CDK1 and PP2A activities change the timing of M-phase entry. This indicates that Xenopus early embryo contains a specific mechanism allowing for fine control of cleavages (El Dika et al., 2014b), which may be important for temporal adjustment of cell divisions in the overall developmental program.

The process that we are describing is multifaceted and very complex. Therefore it is important to formulate a suitable mathematical model that may capture the most important features of this process. While this model certainly must be a simplification of the biological reality, it may deliver detailed information that is often unattainable experimentally (Bellomo \& Carbonaro, 2011).

\section{Results}

Interplay between CDK1 and PP2A modifies timing of M-phase entry: previous experimental data for the mathematical model

Our previous studies described a potential mechanism by which Xenopus laevis embryos could adjust the timing of their cell divisions to the developmental program. Briefly, moderate inhibition of CDK1 with RO3306 (Vassilev et al., 2006) at low concentrations (1 or $2 \mu \mathrm{M}$ ), applied to an embryonic cell-free extract right before entry into the first mitotic M-phase, provokes a dose-dependent delay in M-phase entry as suggested by the state of phosphoryla-

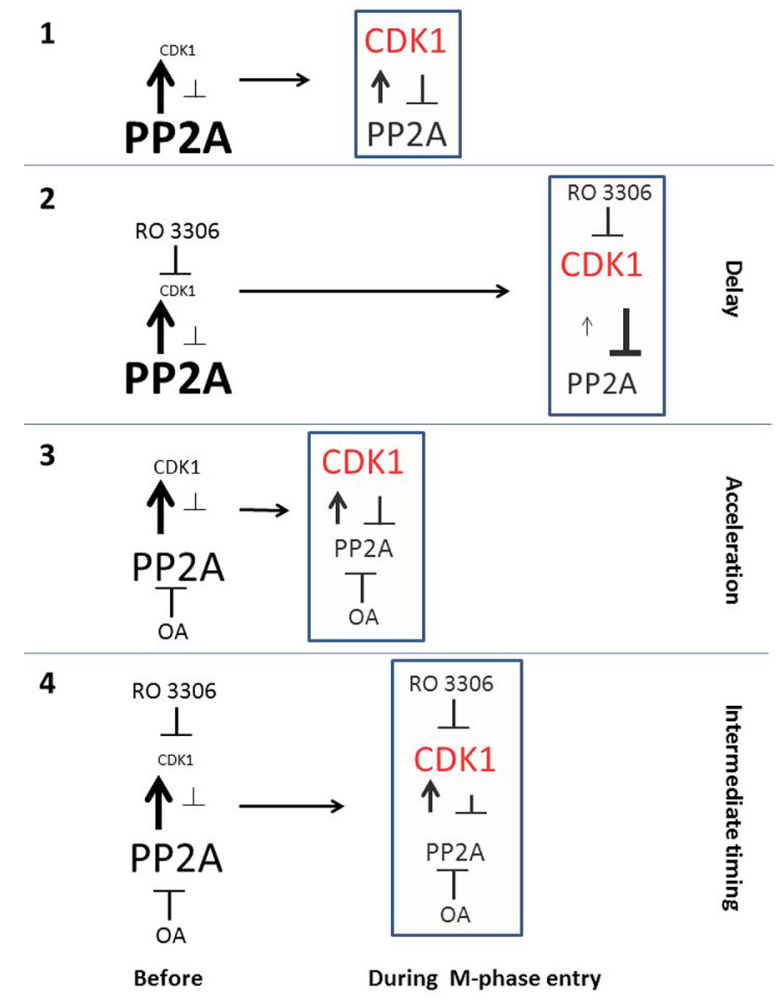


tion of its substrates CDC27 and MCM4 (El Dika et al., 2014b). Conversely, inhibition of PP2A phosphatase by okadaic acid (OA; at 1 or $2.5 \mu \mathrm{M}$ concentration, but not at $200 \mathrm{nM}$ or lower) accelerates the onset of mitosis also in a dose-dependent manner (Fig. 2; Felix et al., 1989; El Dika et al., 2014b).

However, the fact that the lower doses of OA (50-200 nM) do not change the timing of $\mathrm{M}$-phase suggests the relative robustness of the system, which is able to "buffer" the effects of a moderate inhibition of PP2A. Interestingly, when the two inhibitors, at very low doses ( 1 or $2 \mu \mathrm{M}$ of R03306 and 50-200 nM OA), were added together, an intermediate timing of M-phase entry was observed, again in a dose dependent manner (Fig. 3). This indicates the importance of the fine balance between CDK1 and PP2A activity in regulating the timing of mitosis.

\section{Simple mathematical model of the effect of balance between CDK1 and PP2A on M-phase entry}

We consider the following variables

- the concentration of active CDK1 is described by the variable $y_{1}=y_{1}(t)$; whereas inactive CDK1 by $\tilde{y}_{1}=1-y_{1}$,

- the concentration of PP2A is described by the variable $y_{2}=y_{2}(t)$

- the concentration of $\mathrm{OA}$ is assumed to be constant $a_{0}$,

- the concentration of R03306 is assumed to be constant $a_{3}$. We assume

$$
a_{3} \ll \alpha, \quad a_{0} \ll \beta
$$

We propose a simple model describing the interactions between active CDK1 and PP2A in the presence of constant sources of OA and RO3306 that may influence the kinetics of CDK1 and PP2A activities.

The corresponding simple equation reads

A
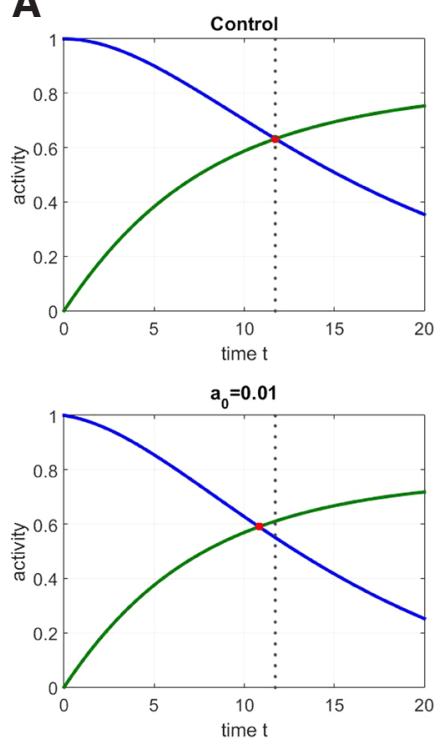
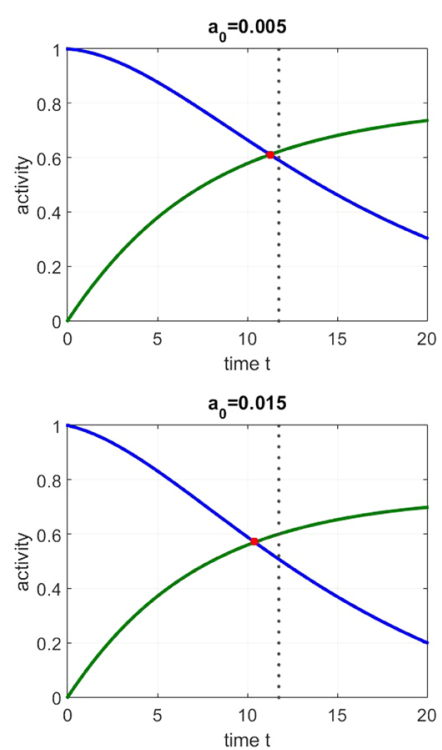

$$
\left\{\begin{array}{c}
\dot{y}_{1}=\alpha \widetilde{y}_{1} y_{2}-a_{3} \\
\dot{y}_{2}=-\beta y_{1} y_{2}-A\left(a_{0}, a_{3}\right) .
\end{array}\right.
$$

where $\alpha, \beta$ are given positive constant coefficients and $A=A\left(a_{0}, a_{3}\right)$ is a function that takes into account the effects of $\mathrm{OA}$ and RO3306 on PP2A. We assume that

$$
\begin{aligned}
& A(0,0)=0, \\
& A\left(a_{0}, 0\right)=a_{0}, \quad a_{0} \geq 0, \\
& A\left(0, a_{3}\right)=0 \quad a_{3} \geq 0, \\
& A\left(a_{0}, a_{3}\right)=\delta_{1} e^{\delta_{2} \ln \left(a_{0}\right)}, \quad a_{0}, a_{3}, \delta_{1}, \delta_{2}>0 .
\end{aligned}
$$

For simplicity we also assume that initially (i.e. for $t=0$ )

$$
y_{1}(0)=0, \quad y_{2}(0)=1
$$

We finally define the time $T$ of entering in mitosis by the condition:

$$
y_{1}(T)=y_{2}(T)
$$

Model Eq. (1) describes the increase in concentration of active CDK1 resulting from the indirect interaction between CDK1 and PP2A and the inhibition of this inhibition by R03306. The decrease in concentration of active PP2A is due to the indirect interaction of PP2A with CDK1, which is inhibited by both OA and R03306. This, in turn, influences the dynamics of CDK1 activity increase. The interaction terms are simplified and described in bilinear terms as the result of concentration of these inhibitors. The function
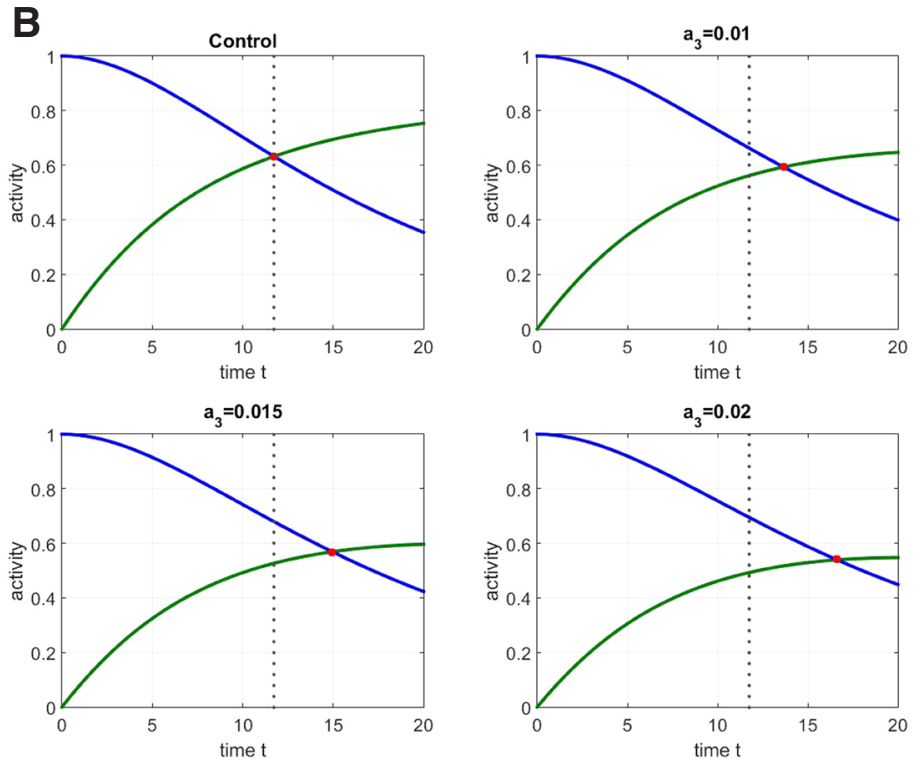

Fig. 4. Simulations showing alterations in M-phase entry. This accelerates when aO increases, i.e. the more OA in the extract, the faster the $M$ phase entry (A). In contrast, it is delayed $\mathbf{w}$ hen a3 increases, i.e. the more RO3306 in the extract, the greater the delay in M-phase entry (B). Blue line, PP2A activity; green line, CDK1 activity; red point, crossing of PP2A and CDK1 activities arbitrarily considered as the time of M-phase entry; dotted line, time of M-phase entry in control. 
describing the inhibition of the decrease of PP2A activity has a discontinuous nature due to its biological interpretation: we apply the value 0 when both $\mathrm{OA}$ and $\mathrm{R} 03306$ are absent; the value of the concentration of $\mathrm{OA}$ when $\mathrm{RO} 3306$ is absent; the value 0 when $\mathrm{OA}$ is absent and the nonzero value when both OA and R03306 are present. In this last case we use the exponent function that, with respect to the first variable, is an increasing and convex function and has a stronger than linear growth close to 0 . In the presence of R03306 (the second variable higher than 0) the function itself is independent of the concentration of $\mathrm{RO} 3306$. We are convinced that such a function reflects biological reality. Fig. 4 and 5 show the simulations issued from the model Eq. (1).

As mentioned in the first section of the Results, low doses of OA did not change the timing of M-phase entry. However, when both inhibitors were added to the extract, an intermediate timing of M-phase entry was observed. We applied our model also to this situation (Fig. 6 A, B, C, D).

This simulation accurately reflects experimental results.
Mathematical model of the balance between CDK1 and PP2A in controlling M-phase entry with integration of CDC25 activity

In our second model we additionally consider the influence of CDC25, which was not addressed in the model (1).

Let the concentration of active CDC25 be described by the variable $y_{5}=y_{5}(t)$; whereas inactive CDC25 by $\tilde{y}_{5}=1-y_{5}$.

The more complex model reads

$$
\left\{\begin{array}{c}
\dot{y}_{1}=\alpha \tilde{y}_{1} y_{2}+\gamma \widetilde{y}_{1} y_{5}-a_{3} \\
\dot{y}_{2}=-\beta y_{1} y_{2}-A\left(a_{0}, a_{3}\right) \\
\dot{y}_{5}=\omega y_{1} \widetilde{y}_{5} .
\end{array}\right.
$$

where $\alpha, \beta, \gamma, \omega, \delta_{1}, \delta_{2}$ are given positive constants coefficients.

For simplicity we may assume that initially (i.e. for $t=0$ ) we have

$$
y_{1}(0)=0, \quad y_{2}(0)=1, \quad y_{5}(0)=0 \text {. }
$$

A
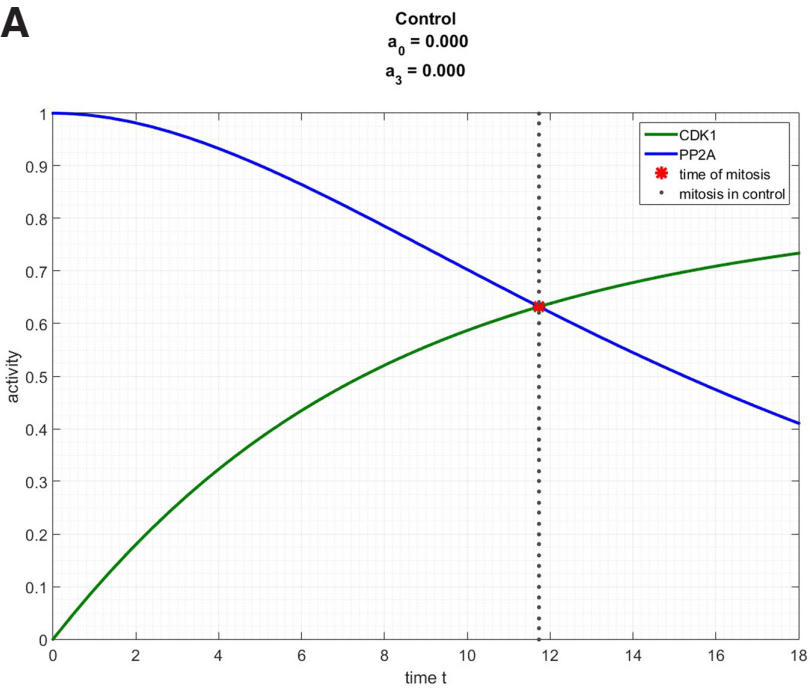

C

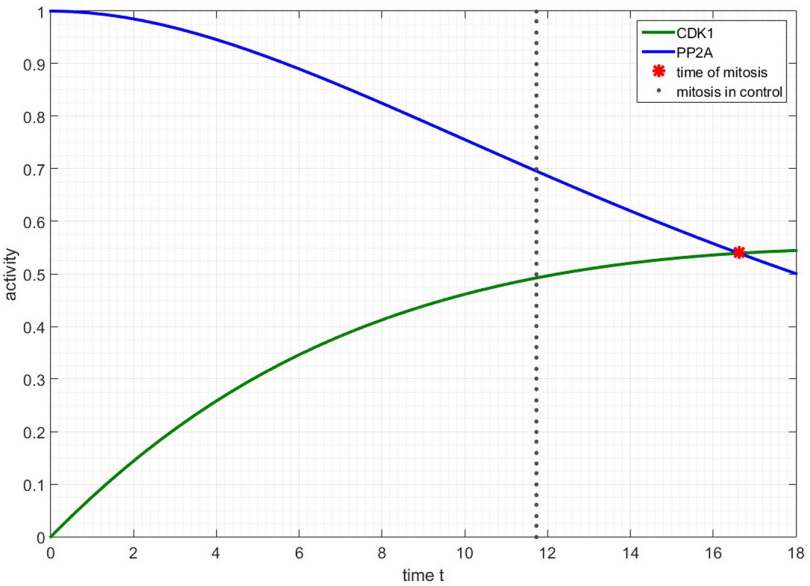

B

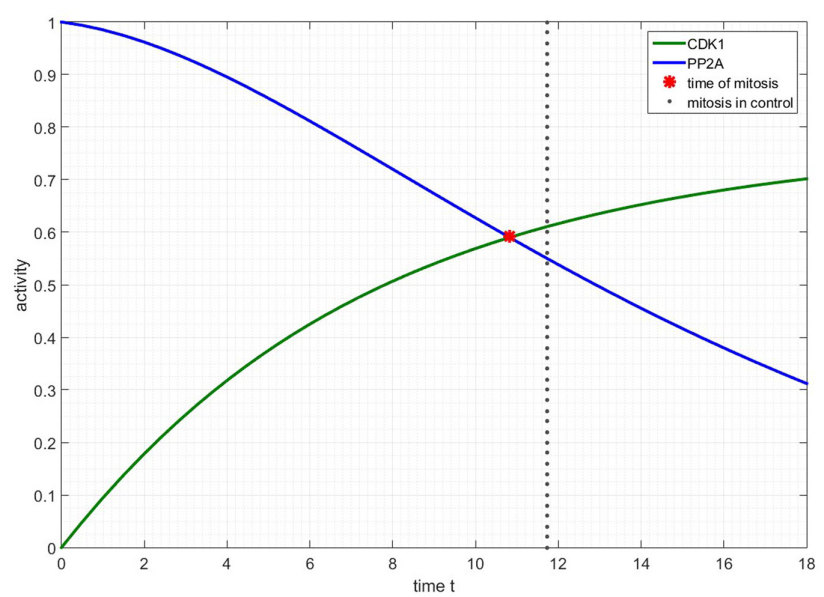

D

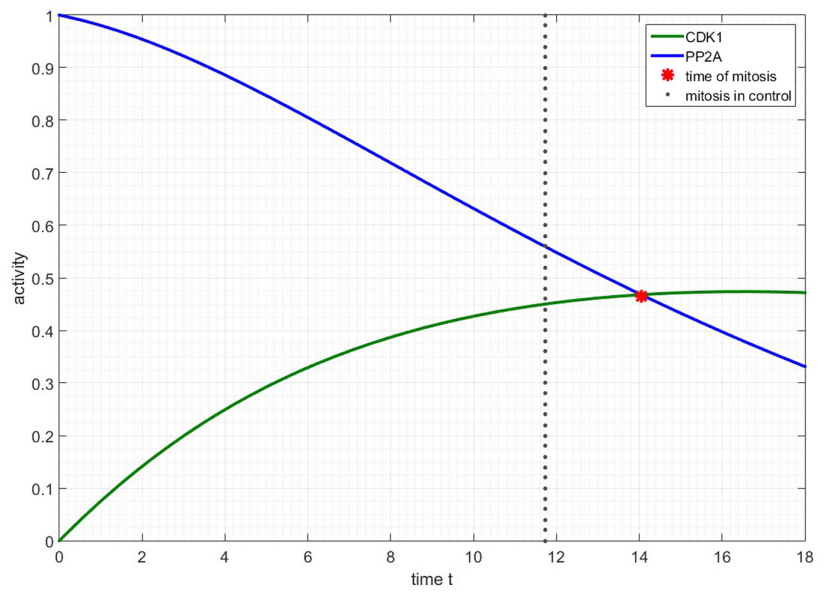

Fig. 5. Simulations showing modifications of the timing of M-phase when the control extract (A) is supplemented with RO3306 (B), OA (C) or R03306+OA (D). When we increase $a_{0}$ to value much higher than 0 (e.g. 0.01), we observe acceleration in M-phase entry independently of parameter $a_{3}$ and the delay when parameter $a_{3}$ increases. 
A
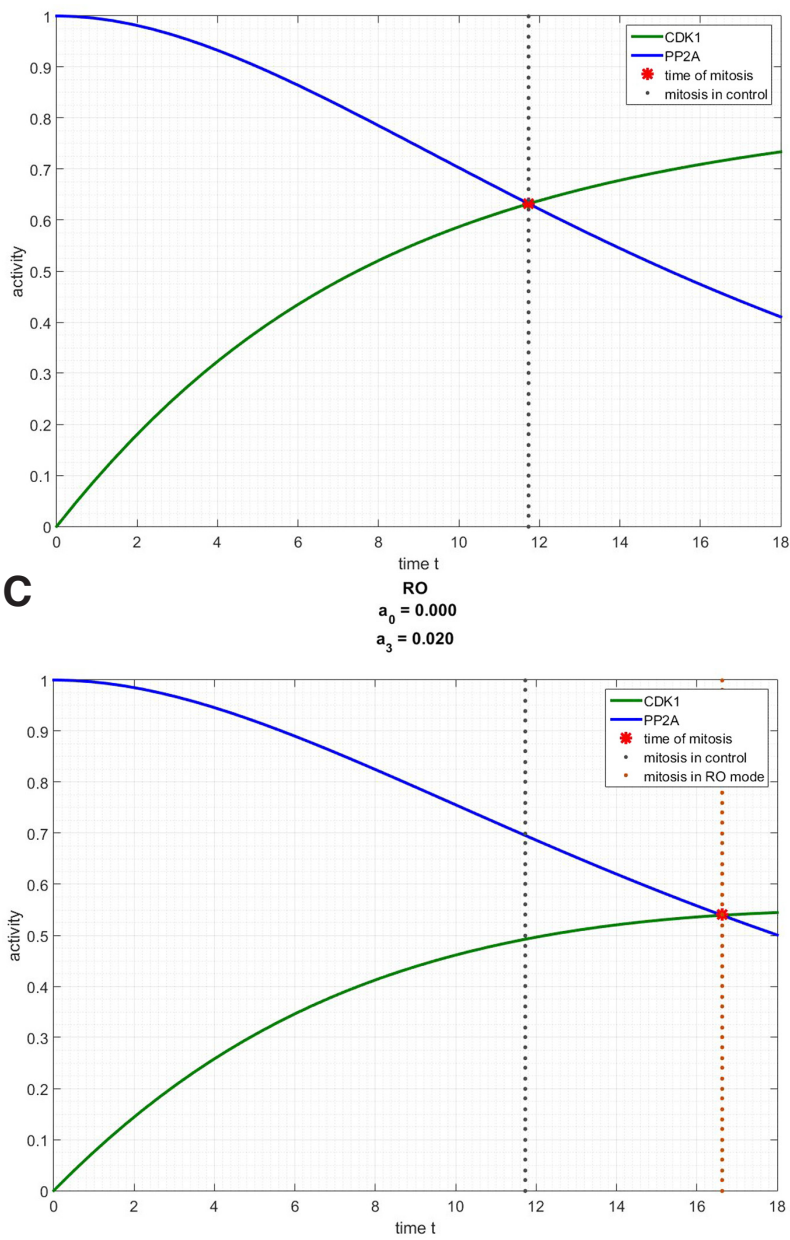

B
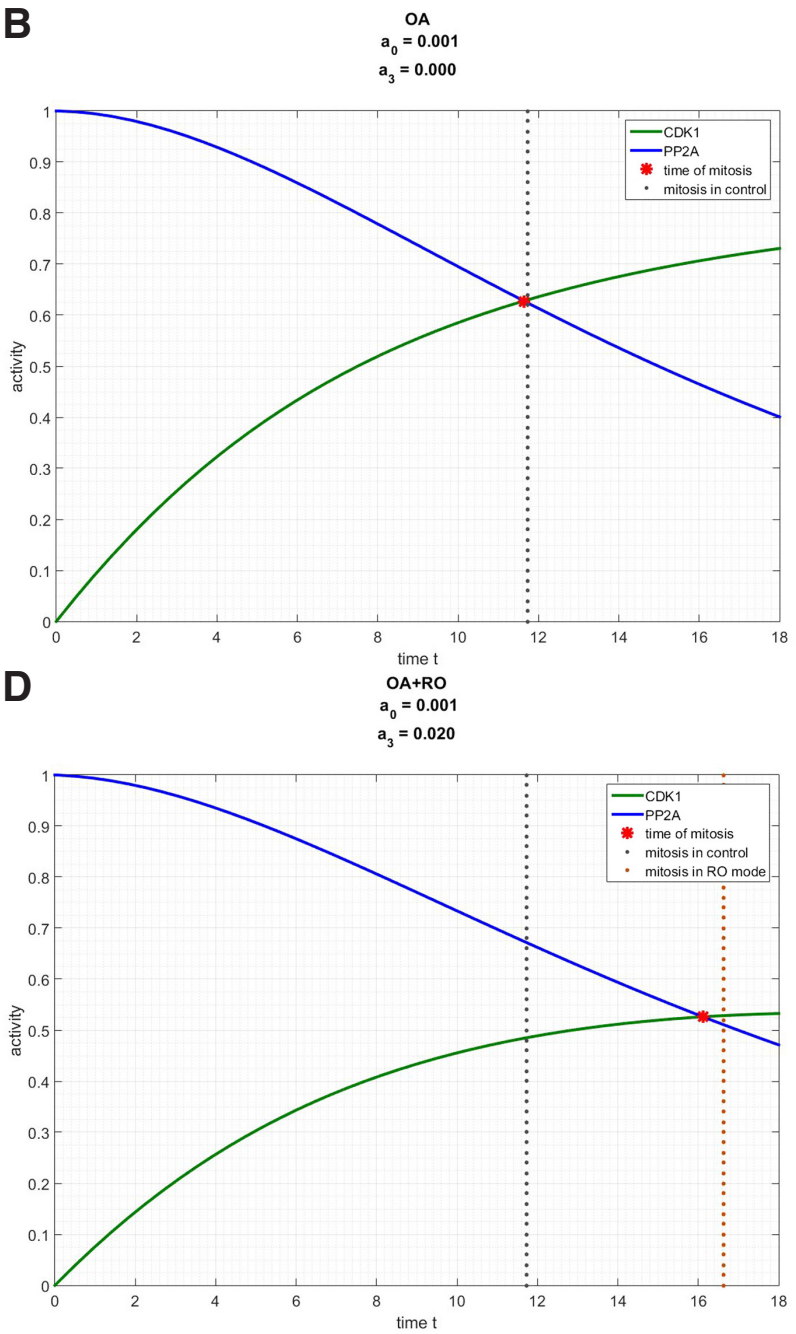

Fig. 6. Simulations showing modifications of the timing of M-phase when the control extract (A) is supplemented with RO3306 alone (B), low dose of OA alone (C) or R03306 in conjunction with low dose of OA (D). When we increase parameter $a_{0}$ to certain low value e.g. 0.01 , there is no change in the timing of entry into $M$-phase for $a_{3}=0$ and the acceleration for parameter $a_{3}>0$.

We assume that the time of entering in mitosis $T$ is defined by the condition:

$$
y_{1}(T)=y_{2}(T)
$$

The model (2), in addition to the parameters incorporated in the model Eq. (1), includes the process of activation of CDC25 (the third equation of (2)) and the effect of active CDC25 on the increase of CDK1 activity.

Simulations of this model (Eq. (2)) gave very similar to those obtained with the simpler model (Eq. (1)) without CDC25 parameter. For simplicity, we show here only one example of these results: Fig. 7 shows the simulation for the interaction between PP2A and CDK1 activities in control and in OA + R03306 in two variants: without and with CDC25 activity.

Analysis of these simulations shows that the time points of Mphase entry do not differ significantly, and the curve in the variant of the model with CDC25 parameter runs closer to the control curve (without inhibitors) than the one without CDC25. Thus, the model including CDC25 shows, as expected, that addition of this parameter increases CDK1 activity and slightly accelerates M-phase entry.

Dynamics of CDC25 during M-phase entry in experimental variants in the presence of RO3306 and OA in the cell-free extract

To verify whether our assumptions concerning the dynamics of CDC25 activation in different experimental conditions was correct, we analyzed the pattern of CDC25 activation in cell-free extracts in control and in the presence of $2 \mu \mathrm{M}$ R03306 and $200 \mathrm{nM}$ OA, or both. We followed the pattern of CDC25 phosphorylation by Western blot, which reflects its activity.

As shown in Fig. 8B, the dynamics of CDC25 phosphorylation/ activation are very similar in the control and in the presence of $2 \mu \mathrm{M}$ R03306 and 200 nM OA separately. However, when the two inhibitors were present simultaneously, a slight initial delay in CDC25 activation was observed. In this case the levels of CDC25 phosphorylation became equal for all variants within $16-20 \mathrm{~min}$ of incubation.

These results prompted us to modify the dynamics of CDC25 


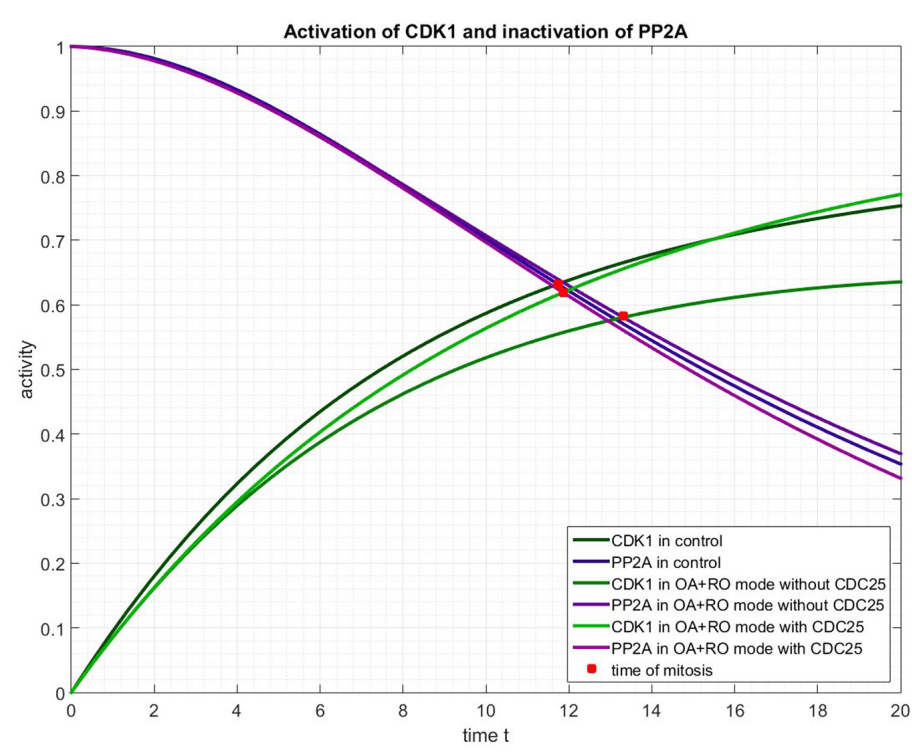

Fig. 7. Simulations showing the time of M-phase entry as a result of interactions between PP2A and CDK1 in control and in $\mathrm{OA}+\mathrm{RO} 3306$ with and without $C D C 25$ function.

activation in our model for the variant of $\mathrm{OA}$ and $\mathrm{RO} 3306$.

\section{Mathematical model of balance between CDK1 and PP2A on $M-p h a s e$ entry taking in the account the delayed activation of CDC25}

In the case where we consider the delay in CDC25 activation we implemented $\gamma=\gamma(t)$ where $\gamma$ is a switch-like function from value 0 to a positive value $\psi$. We consider two cases, when $\gamma(t)$ is continuous $\left(y(t)=\gamma_{1}(t)\right.$ ) and discontinuous (impulsive, $\gamma(t)=\gamma_{2}(t)$ ).

The model has the following form

$$
\left\{\begin{array}{c}
\dot{y}_{1}=\alpha \tilde{y}_{1} y_{2}+\gamma_{i}(t) \tilde{y}_{1} y_{5}-a_{3}, \\
\dot{y}_{2}=-\beta y_{1} y_{2}-A\left(a_{0}, a_{3}\right), \\
\dot{y}_{5}=\omega y_{1} \tilde{y}_{5},
\end{array}\right.
$$

for $i=1,2$, where $\gamma_{1}(t)$ is the form of continuous case for the function $\gamma(t)$ defined as

$$
\gamma_{1}(t)=\left\{\begin{array}{c}
0, \quad t<\tau, \\
\psi \cdot \frac{t-\tau}{t-\tau+1}, \quad t \geq \tau .
\end{array}\right.
$$

and $\gamma_{2}(t)$ is the form of discontinuous case for the function $\gamma(t)$ defined as

$$
\gamma_{2}(t)= \begin{cases}0, & t<\tau, \\ \psi, & t \geq \tau,\end{cases}
$$

for positive constants $\psi, \tau$.

In Fig. 9A, B, C, D we show the simulations of these three situations: 9A) when the activity of CDC25 rises similarly in all four experimental variants, $9 \mathrm{~B}$ ) when it is slightly delayed in $\mathrm{OA}$ and $\mathrm{RO} 3306$ variant and is represented by continuous function, $9 \mathrm{C})$ when it is slightly delayed in $\mathrm{OA}$ and $\mathrm{RO} 3306$ variant and is represented by impulsive function, and 9D) all three variants and the control are superposed for direct comparison.

In comparison with the model Eq. (2) - the model (3) allows the time varying (e.g. impulsive) coefficient defining the influence of CDC25 on the increase in CDK1 activity.

Simulations of our third model with CDC25 activity (Eq. (3)) are very similar to the simulations of the second model (Eq. (2)) in all variants. For simplicity, we compare only CDK1, PP2A activities and the resulting times of $\mathrm{M}$-phase entry in $\mathrm{OA}+\mathrm{RO} 3306$ containing variants with non-delayed and delayed CDC25 activation represented by continuous and impulsive function (Fig. 10). Thus, we conclude that the delay in CDC25 activation slightly delays the timing of M-phase entry.

\section{Dynamics of CDK1 dephosphorylation on tyrosine 15 during M-phase entry in experimental variants in the presence of RO3306 and OA in cell-free extract}

CDK1 activation correlates with its dephosphorylation on threonine 14 and tyrosine 15 (Tyr15) by the CDC25 phosphatase (Gould \& Nurse, 1989; Gautier et al., 1991). Thus, the Western blot with anti-Tyr15-phosphorylated CDK1 antibody showing the decrease of its intensity should mirror CDC25 activation. However, as shown in fig. 11, the dynamics of CDK1 Tyr15 dephopshorylation did not
A

$$
\begin{array}{lllllll}
4 & 8 & 12 & 16 & 20 & 24 & 28 \\
\end{array}
$$

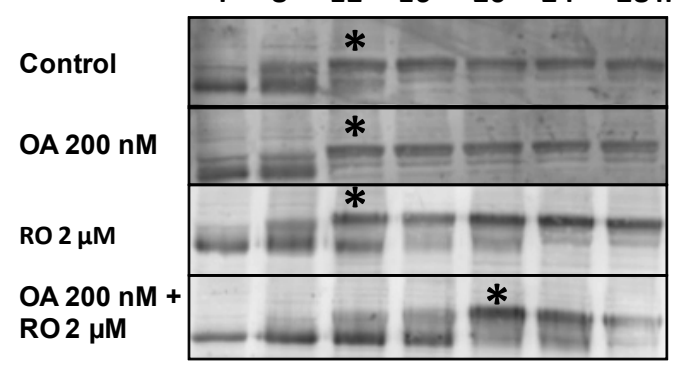

\section{B $\quad$ P-CDC25\%}

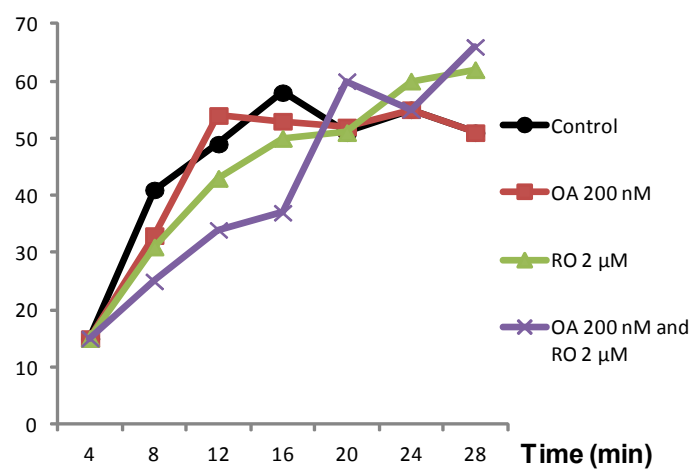

Fig. 8. Dynamics of CDC25 phosphorylation. (A) Western blot of CDC25 in four experimental variants showing the dynamics of CDC25 phosphorylation. The dynamics are very similar in the control and in the presence of $O A$ and $R O$ separately, but CDC25 phosphorylation was slightly delayed when the two inhibitors were present simultaneously. Note that the timing of M-phase entryin the $O A+R O$ variant is accelerated in comparison to the presence of $R O$ alone in the extract, despite the initial delay in CDC25 activation. The asterisks show the time points when the majority of CDC25 becomes phosphorylated (the most up-shifted band). The loading control was Ponceau $S$ red staining of the membrane (not shown). (B) Graph showing the dynamics of the increase in the hyperphosphorylated form of CDC25 (fully active CDC25). Note that in the variant with OA+RO, the curve is initially delayed and joins other curves between 16 and 20 min. 
show significant difference between the control, RO3360 and OA variants in comparison to $\mathrm{OA}+\mathrm{RO} 3360$ variant, in which $\mathrm{CDC} 25$ activation was slightly delayed. Fig. 11 shows such a Western blot.

Thus, the delay in CDC25 activation in the presence of R03360+0A seems to be balanced probably by increased Myt1/ Wee1 kinase(s) activity. The timing of M-phase entry in all four variants seems to correlate with the initial dynamics of CDK1 Tyr15 dephosphorylation: both are the fastest in the presence of $\mathrm{OA}$, lower in control, even lower in the presence of $\mathrm{OA}+\mathrm{RO}$ and the lowest in the presence of $\mathrm{RO}$ alone.

\section{Discussion}

The goal of our mathematical model of the interplay between CDK1 and PP2Aactivities in determining the timing of M-phase entry was to verify whether the experimental data obtained previously (EI
Dika et al., 2014b) can be described in mathematical terms, and whether the analysis of simulations can predict how the regulatory networks determine the dynamics of these activities and the control of cell cycle events. These two goals were fully achieved.

Here we present a simple model accurately emulating the interplay between CDK1 and PP2A activities in determining the timing of the M-phase entry. We add further complexity to this model by integrating the dynamics of the CDC25 phosphatase, which is the major activator of CDK1 at mitosis. For simplicity, we arbitrary designated the intersection of CDK1 and PP2A curves as the Mphase onset time point. In reality, this time point is determined by the minimal level of CDK1 activation that is sufficient to trigger significant phosphorylation of its critical substrates. The accumulation of substrates phosphorylated by CDK1 and dephosphorylated by PP2A is escalating and dynamic; thus, even in biological experiments, we have to arbitrarily choose the time point of the M-phase
A

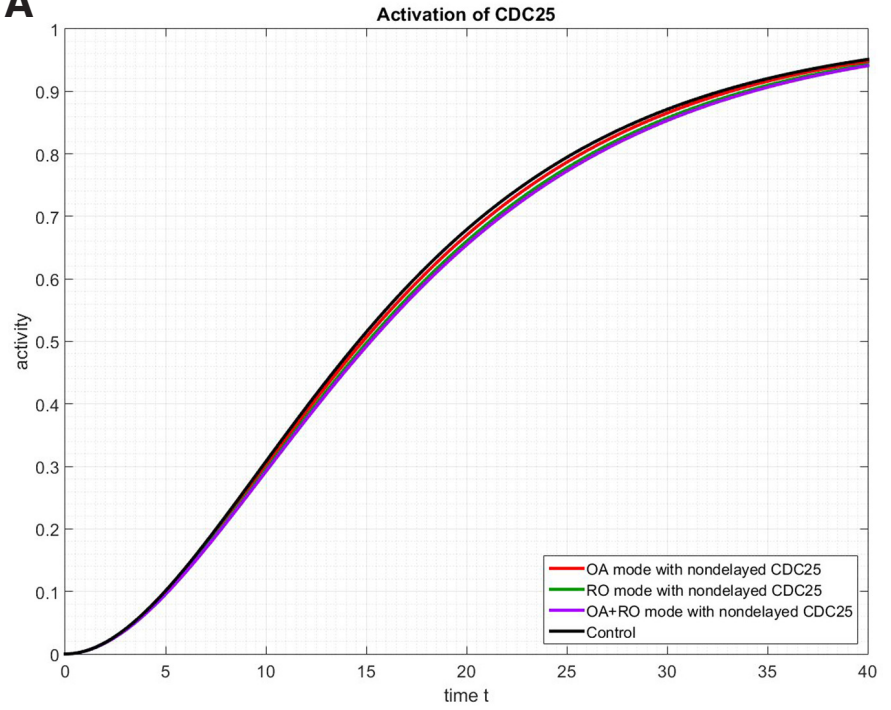

C

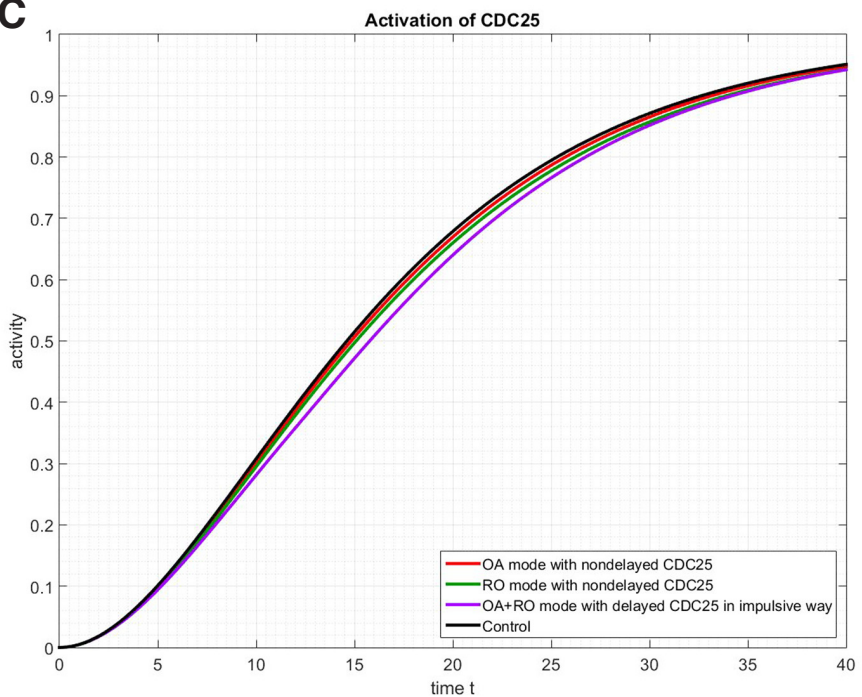

B
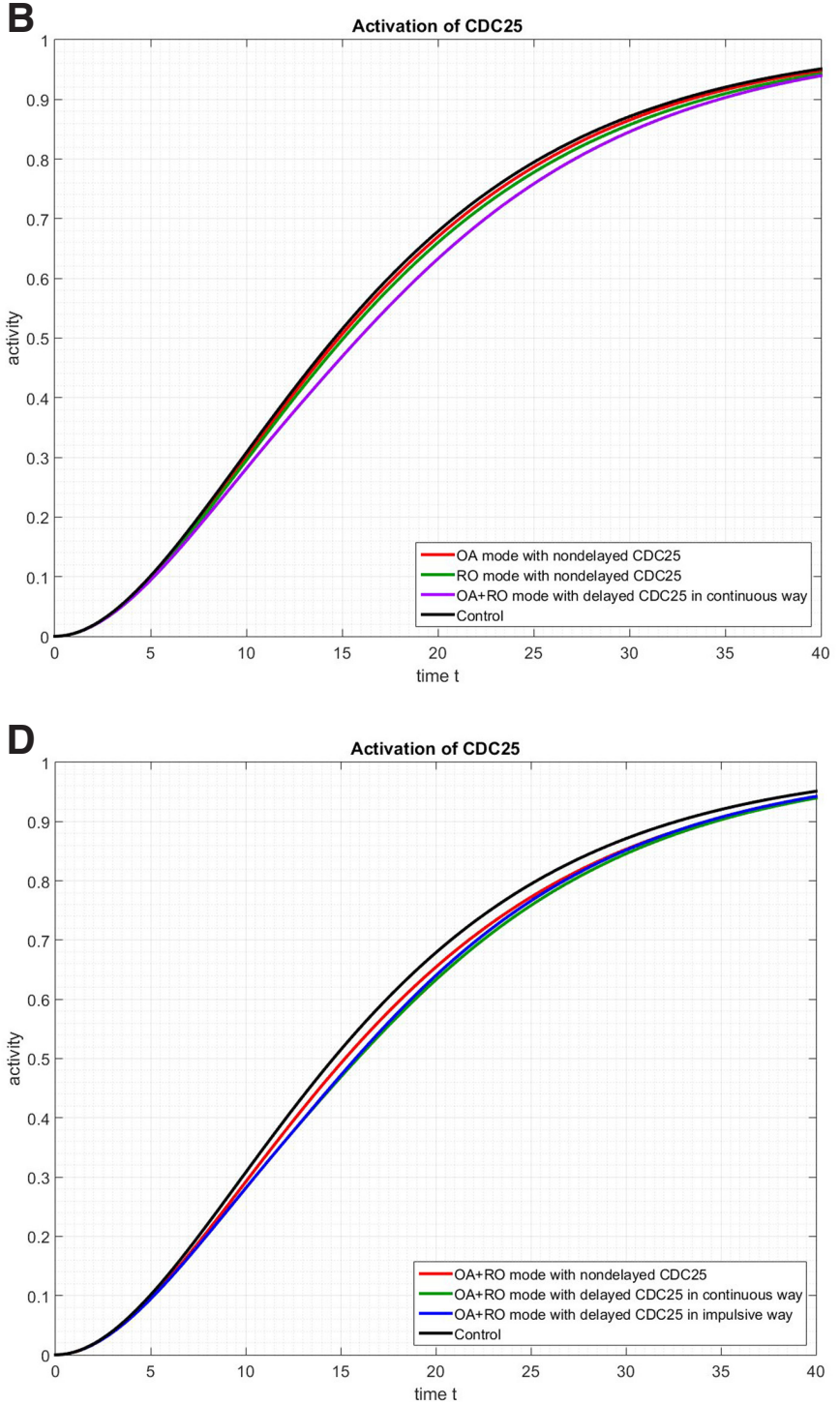

Fig. 9. Simulations of CDC25 activity dynamics. (A) CDC25 activity rises similarly in all four experimental variants. (B) It is slightly delayed in the OA and $R 03306$ variant and represented by a continuous function. (C) CDC25 activity is slightly delayed in the OA and RO3306 variant and is represented by an impulsive function. (D) All three variants and the control are superposed for direct comparison. 
A

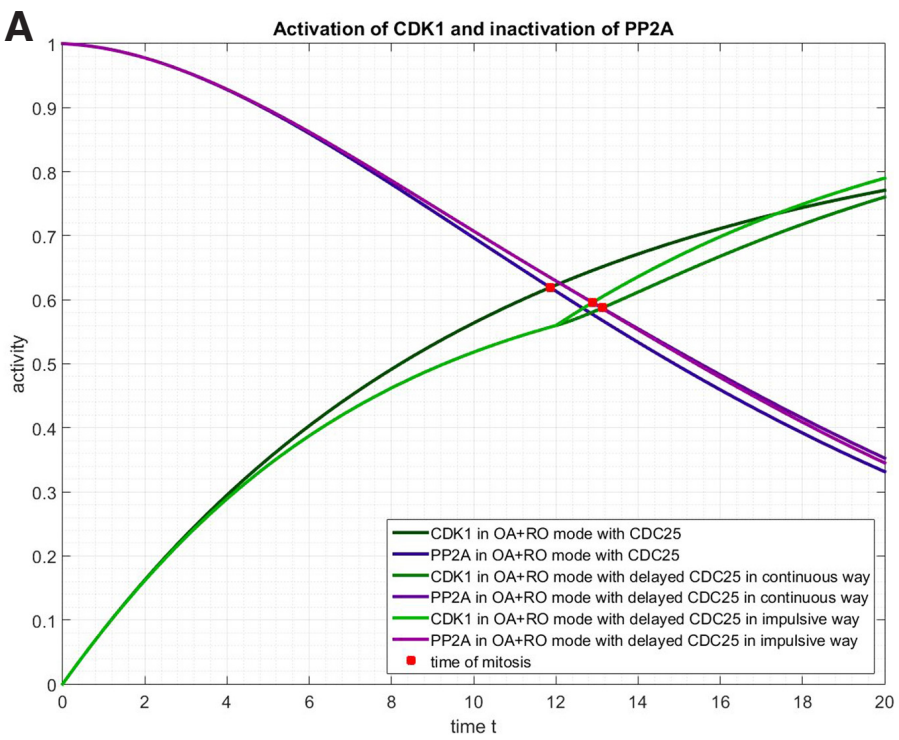

B

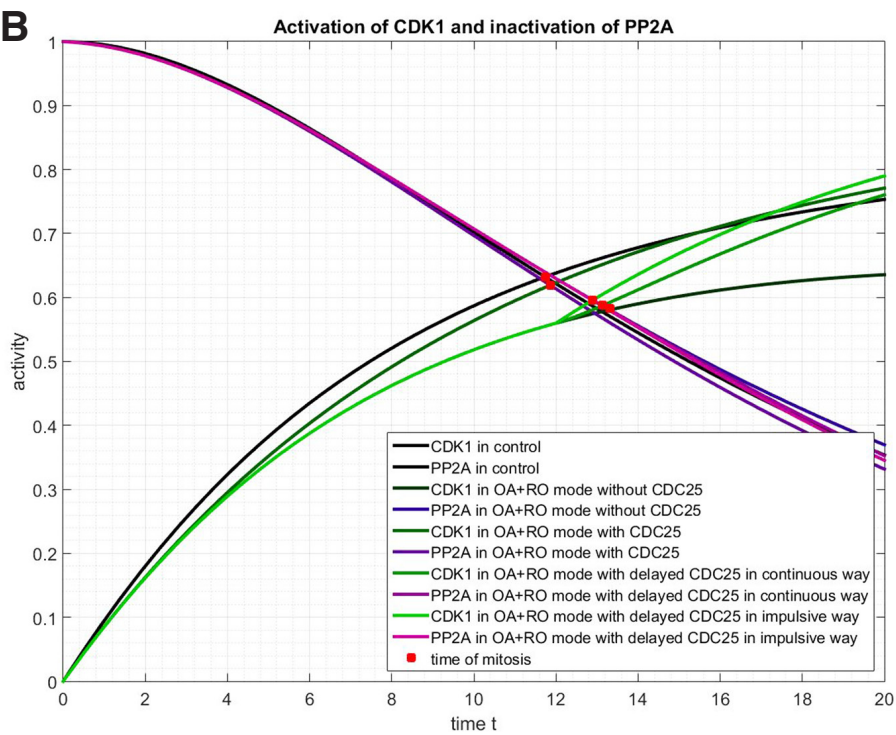

Fig. 10. Simulations showing the time of M-phase entry. Simulations showing the time of M-phase entry as a result of interactions between PP2A and CDK1 in $O A+R O$ with non-delayed and delayed activation of CDC25 represented by continuous and impulsive function (A), and the summary of all variants for $O A+R O(\mathbf{B})$.

entry (El Dika et al., 2014b).

The simulations presented in this paper show how inhibitors of CDK1 and PP2A (RO3306 and OA respectively) modify the curves and in consequence the timing of $\mathrm{M}$-phase entry in all experimental variants, i.e. in extracts with $\mathrm{OA}, \mathrm{RO}$ and $\mathrm{RO}+\mathrm{OA}$ (Fig. 4 and 5). We also managed to emulate in our simulations the experimental data showing that minimal doses of OA do not provoke significant changes in the time of M-phase entry, while it is amplified when OA is combined with $\mathrm{RO}$ (Fig. 6). These results confirm the accuracy of our mathematical model.

The addition of the CDC25 parameter in our model slightly accelerated M-phase entry, but did not change significantly the dynamics of curves of CDK1 activation and PP2A inactivation (for $\mathrm{OA}+\mathrm{RO}$ see Fig. 7). Moreover, the addition to our model of a delayed dynamics of CDC25 activation, which we found by Western blotting in the variant of cell-free extract incubated with $\mathrm{OA}+\mathrm{RO}$, slightly delays the $\mathrm{M}$-phase entry. This is not surprising because any delay in CDC25 must slow down the dynamics of CDK1 activation. However, impulsive vs. continuous mode of CDC25 delay in the $\mathrm{OA}+\mathrm{RO}$ group does not change the timing significantly (see fig. 10). Thus, the simple model seems to emulate correctly the whole process in which the interplay between CDK1 activation and PP2A inactivation determines the timing of M-phase entry. The more complex models that include the $\mathrm{CDC} 25$ parameter probably show more accurately the dynamics of the analyzed processes, but do not change significantly their mathematical description.

Surprisingly, the dynamics of phosphorylation of CDC25 do not mirror the dynamics of CDC25-induced dephosphorylation of CDK1 on tyrosine 15. Our experimental OA+RO variant showed that the delay in $\mathrm{CDC} 25$ phosphorylation did not translate into a
A

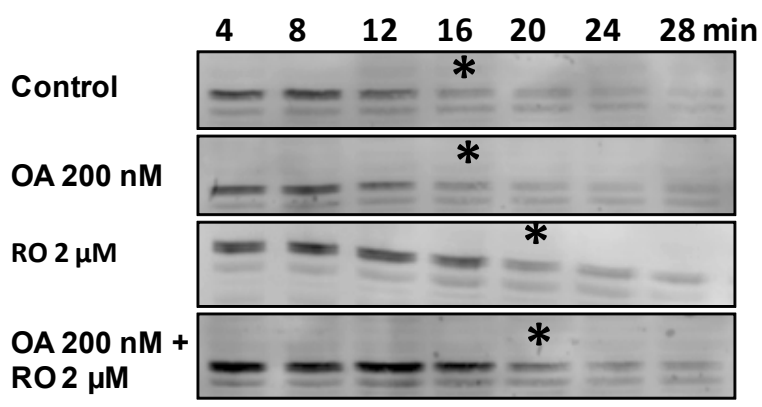

B

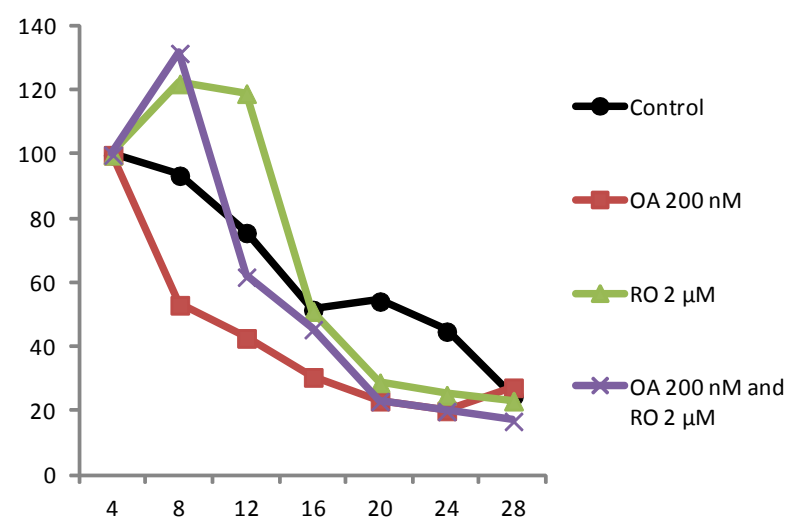

Fig. 11. Dynamics of CDK1 Tyr15 dephopshorylation. (A) Western blot of P-Tyr15 CDK1 from four experimental variants shows that the dynamics of CDK1 dephosphorylation on tyrosine 15 are very similar in the control and in the presence of OA, but are slightly delayed in the presence of RO alone and when the two inhibitors are present simultaneously. The asterisks show the time points when the CDK1 phospho-Tyr15 is visibly reduced. The loading control was Ponceau S red staining of the membrane (not shown). (B) Dynamics of the decrease of P-Tyr15 CDK1. Note that in the presence of $R O(R O$ alone and $R O+O A)$, the curves of P-Tyr15 CDK1 initially increase and fall down to the same level as in the control and OA at the 12 and 16 min time points. 
delay in overall dephosphorylation of CDK1 tyrosine 15 (see Fig. 11), and hence in activation of CDK1. The slightly delayed activation of CDC25 in the presence of OA + RO is probably compensated by Wee1/Myt 1 pathway, which positively controls CDK1 tyrosine 15 phosphorylation (Fattaey \& Booher, 1997).

We also observed that the addition of RO alone or in combination with $O A$ induces a very rapid but moderate increases in phosphorylation of CDK1 tyrosine 15 in the extract (see Fig. 11). It seems that $\mathrm{RO}$, if applied right before the M-phase entry, somehow enhances the Wee1/Myt1 pathway. It Is possible that the addition of RO inhibits traces of activity of CDK1 present in the extract at this particular time point preceding M-phase entry, and this in turn transiently stimulates the Wee1/Myt1 pathway. In consequence, CDK1 tyrosine 15 dephosphorylation preceding M-phase entry begins from a slightly higher level in the presence of RO than in its absence. However, this effect ceases very rapidly and the dynamics of CDK1 tyrosine 15 dephosphorylation becomes similar in all experimental groups. The slightly increased phosphorylation of CDK1 tyrosine 15 (triggering presumed inactivation of traces of CDK1 activity and up regulation of Wee1/Myt1 pathway) results in the delay of $C D C 25$ phosphorylation in the $\mathrm{OA}+\mathrm{RO}$ variant through a feedback mechanism in which CDK1 stimulates CDC25 activation (Fattaey \& Booher, 1997). This explanation seems plausible because also in the variant with the RO alone, CDC25 phosphorylation is slightly delayed (see the green curve in Fig. $8 \mathrm{~B}$ and compare positions of asterisks in Fig. 8A), but less than in the $\mathrm{OA}+\mathrm{RO}$ variant (compare with the mauve curve in Fig. 8B). Nevertheless, the timing of M-phase entry seems to correlate well with the timing of the initial CDK1 dephosphorylation on tyrosine 15. This suggests that the delay in M-phase entry in the presence of $\mathrm{RO}(\mathrm{RO}$ alone and $\mathrm{OA}+\mathrm{RO})$ results from the initial increase of CDK1 Tyr 15 phosporylation preceding M-phase entry. As confirmed by our mathematical model, the further increase in CDK1 activity can be partially balanced by PP2A. For example: a relatively lower $C D K 1$ activity in the presence of $R O$ in comparison to the control can be balanced by slower inactivation of PP2A resulting in the delay in CDK1 substrates dephosphorylation and effectively determining the time of M-phase entry. Thus, our data suggest that the regulation of the whole system strives, through the feedbacks regulating cell cycle enzymes, to achieve a state similar to the conditions in the control extract.

In conclusion, the data presented in this paper confirm that our simple mathematical model accurately emulates experimental results showing that the interplay between CDK1 activation and PP2A inactivation determines the timing of M-phase entry in Xenopus laevis embryo cell-free extract. The incorporation of CDC25 activity dynamics as a parameter in our model emulates more precisely the experimental data, but does not significantly change the simulations. The incorporation into our model of the experimentally demonstrated delay in the dynamics of CDC25 activation in the case of simultaneous presence of OA and RO confirmed a slight delay in the M-phase entry. These results strengthen our hypothesis that Xenopus laevis embryos possess certain flexibility in their cell cycle regulation despite the tendency to robustly control the timing of cell cycle events. The balance between CDK1 and PP2A activities seems to play a crucial role in this regulation, but the timing of M-phase entry is certainly determined by the whole complex regulatory network, for which a number of components are still unknown.

\section{Materials and Methods}

\section{Mathematical analysis}

We propose two mathematical models: one includes the interaction between CDK1 and PP2A taking into account the presence of OA and RO3306 and the second includes the activity of CDC25. The models are systems of two equations with two unknown functions describing the temporal behavior of the concentrations of CDK1 and PP2A. The coefficients may depend on time and therefore the behavior of the solutions may be complex. As usual in the case of any mathematical modeling, the mathematical structure may capture only some aspects of the biological process, but on the other hand may give a more precise picture of the biological process. This apparent paradox is because the mathematical model, while omitting aspects of the biology, provides a better quantitative and dynamic understanding of the studied phenomenon. The methods are based on the study of corresponding dynamical systems, both on analytical and numerical levels. Although the system is quite compact and its mathematical analysis is complex, the biological importance is in numerical solutions that indicate their possible behavior. In particular we are interested in estimation of the time that is needed to enter in mitosis. The problem may be seen as a problem of controlling that time. Simulations were performed using Matlab (version 2015a) software. Table 1 summarizes the parameters used in the simulations.

\section{Egg collection and activation}

Xenopus laevis females were purchased from NASCO (Fort Atkinson, WI, USA). Females were injected subcutaneously with Human Chorionic Gonadotropin HCG (500 IU per female) and kept overnight at $21^{\circ} \mathrm{C}$ in 110 $\mathrm{mM} \mathrm{NaCl}$. Cell-free extracts were prepared as previously described by Murray (1991) using modifications described by Chesnel and colleagues (2005). Metaphase-arrested Xenopus eggs, collected from overnight spawning after HCG injection, were dejellied with $2 \%$ L-cysteine $\mathrm{pH} 7.81$ in XB buffer (100 mM KCl, $1 \mathrm{mM} \mathrm{MgCl} 2,50 \mathrm{mM} \mathrm{CaCl} 2,10 \mathrm{mM}$ HEPES, and 50 $\mathrm{mM}$ sucrose $\mathrm{pH}$ 7.6). Dejellied eggs were washed in XB buffer, activated with $0.5 \mathrm{mg} / \mathrm{ml}$ of calcium ionophore A23187 until cortical contraction was observed, and then extensively washed in XB.

\section{Cell free extracts}

Cytoplasmic extracts from calcium ionophore-activated embryos before the first embryonic mitosis were prepared according to Murray (1991). Embryos were cultured at $21^{\circ} \mathrm{C}$ in XB Buffer for 60-70 minutes postactivation, i.e. until the late first interphase as described previously (Chesnel et al., 2005). They were then transferred into $5 \mathrm{~mL}$ ultraclear ${ }^{\mathrm{TM}}$ centrifuge tubes (Beckman Coulter, Roissy, France) and incubated at $4^{\circ} \mathrm{C}$ in $0.5 \mathrm{~mL}$ of $\mathrm{XB}$ buffer containing protease inhibitors $(10 \mu \mathrm{g} / \mathrm{ml}$ of each aprotinin, leupeptin, pepstatin, chymostatin, and $0.1 \mathrm{mM} \mathrm{AEBSF}$ ) and $25 \mathrm{mg} / \mathrm{ml}$ of cytochalasin D. Subsequently, embryos were subjected to three consecutive centrifugations: first short spin to remove XB excess and pack the embryos in the tube, second $10,000 \mathrm{~g}$ spin at $4^{\circ} \mathrm{C}$ for 10 minutes to separate the embryo fractions, and final $10,000 \mathrm{~g}$ clarification spin of the supernatant at $4^{\circ} \mathrm{C}$ for 10 minutes. The supernatant was then incubated at $21^{\circ} \mathrm{C}$. Every 4 minutes,

TABLE 1

\section{A SUMMARY OF THE PARAMETERS USED IN THE SIMULATIONS}

\begin{tabular}{cc} 
Parameter & Value \\
\hline$\alpha$ & 0.1 \\
$\beta$ & 0.1 \\
$\gamma$ & 0.1 \\
$\delta_{1}$ & 0.75 \\
$\delta_{2}$ & 0.5 \\
$\omega$ & 0.1 \\
$\psi$ & 0.16 \\
$\tau$ & 12 \\
\hline
\end{tabular}




\section{M. Debowski et al.}

aliquots were taken out and directly mixed with Laemmli buffer for Western blot analyses (Laemmli et al., 1970). All aliquots were stored at $-80^{\circ} \mathrm{C}$.

\section{Inhibitors}

RO 3360 (RO), a selective CDK1 inhibitor (Vassilev et al., 2006) was purchased from Merck Millipore. It was used in 1, 2, 4 and $10 \mu \mathrm{M}$ concentrations. The stock solution was dissolved in ethanol. Okadaic acid (OA), from Molecular Probes, known to inhibit both PP2A (in low concentrations of 50-200 nM) and PP1 phosphatase (in high concentrations of 1-2.5 $\mu \mathrm{M}$ ) (Felix et al., 1990) was purchased from Sigma. The stock solution was dissolved in DMSO. We used the low doses of OA (200, 100 and $50 \mathrm{nM})$ in the majority of our experiments because these doses do not change the timing of M-phase entry and partially inhibit PP2A (Felix et al., 1990).

\section{Western blot and antibodies}

Protein extracts were subjected to $8 \%$ sodium dodecyl sulfate polyacrylamide gel electrophoresis (SDS-PAGE) (Laemmli et al., 1970). Separated proteins were transferred to nitrocellulose membranes (Hybond C, Amersham Biosciences). Membranes were blocked with PBS containing 5\% non-fat dry milk and $0.1 \%$ Tween 20 and probed with primary antibodies against Xenopus laevis CDC25 (gift from T. Lorca and A. Castro) and Phospho-Tyr15 CDK1 (Cell Signaling). Antigen-antibody complexes were visualized using alkaline phosphatase-conjugated antirabbit antibodies. The chemifluorescence was detected with the ECF Reagent (Amersham Biosciences). Signal quantification was performed using ImageQuant 5.2 software (Amersham Biosciences).

\section{Acknowledgements}

MED was supported by the Lebanese Ministry of Education. We are grateful to Thierry Lorca and Anna Castro (CRBM, Montpellier, France) for providing us the antibody against CDC25 and to Jenny Wu and Damien Coudreuse (IGDR, Rennes, France) for valuable discussions and corrections of the manuscript.

\section{References}

BELLOMO N, CARBONARO B. (2011). Toward a Mathematical Theory of Living Systems focusing on Developmental Biology and Evolution: A Review and Perspectives Phys. Life Rev. 8: 1-18

COUDERAUSE D, NURSE P. (2010). Driving the cell cycle with a minimal CDK control network. Nature 468: 1074-1079.

ELDIKAM, LASKOWSKA-KASZUBK, KORYTOM, DUDKAD, PRIGENTC, TASSAN JP, KLOC M, POLANSKI Z, BORSUK E, KUBIAK JZ. (2014a). CDC6 controls dynamics of the first embryonic M-phase entry and progression via CDK1 inhibition. Dev Biol. 396: 67-80.

EL DIKA M, DUDKA D, PRIGENT C, TASSAN JP, KLOC M \& KUBIAK JZ. (2014b). Control of timing of embryonic M-phase entry and exit is differentially sensitive to CDK1 and PP2A balance. Int. J. Dev. Biol. 58: 767-774.

FATTAEY A, BOOHER RN. (1997). Myt1: a Wee1-type kinase that phosphorylates Cdc2 on residue Thr14. Prog Cell Cycle Res. 1997: 233-240.

FELIX MA, PINES J, HUNT T, KARSENTI E. (1989). Temporal regulation of cdc2 mitotic kinase activity and cyclin degradation in cell-free extracts of Xenopus eggs. J Cell Sci Suppl.12: 99-116.

FISHER D, KRASINSKA L, COUDREUSE D, NOVAK B.(2012). Phosphorylation network dynamics in the control of cell cycle transitions. J Cell Sci. 125: 4703-4711.

GAUTIER J, SOLOMON MJ, BOOHER RN, BAZAN JF, KIRSCHNER MW. (1991) cdc25 is a specific tyrosine phosphatase that directly activates p34cdc2. Cell 67: 197-211.

GOULD KL, NURSE P. (1989). Tyrosine phosphorylation of the fission yeast cdc2+ protein kinase regulates entry into mitosis. Nature 342: 39-45.

HARTLEY RS, REMPEL RE, MALLER JL. (1996). In vivo regulation of the early embryonic cell cycle in Xenopus. Dev Biol. 173: 408-419.

HUNTT. (2013). On the regulation of protein phosphatase $2 A$ and its role in controlling entry into and exit from mitosis. Adv Biol Regul. 53: 173-178.

KUMAGAI A, DUNPHY WG. (1991). The cdc25 protein controls tyrosine dephosphorylation of the cdc2 protein in a cell-free system. Cell. 64: 903-914.

LORCA T, CASTRO A. (2013). The Greatwall kinase: a new pathway in the contro of the cell cycle. Oncogene 32: 537-543.

MOCHIDAS, HUNT T. (2012). Protein phosphatases and their regulation in the control of mitosis. EMBO Rep. 13: 197-203.

VASSILEV LT, TOVAR C, CHEN S, KNEZAVIC D, ZHAO X, SUN H, HEIMBROOK DC, CHEN L. (2006). Selective small-molecule inhibitor reveals critical mitotic functions of human CDK1. Proc Natl Acad Sci USA 103: 10660-10665. 


\section{Further Related Reading, published previously in the Int. J. Dev. Biol.}

Control of timing of embryonic M-phase entry and exit is differentially sensitive to CDK1 and PP2A balance Mohammed El Dika, Damian Dudka, Claude Prigent, Jean-Pierre Tassan, Malgorzata Kloc and Jacek Z. Kubiak Int. J. Dev. Biol. (2014) 58: 767-774

Identification of the sperm motility-initiating substance in the newt, Cynops pyrrhogaster, and its possible relationship with the acrosome reaction during internal fertilization

Toshihiko Watanabe, Hideo Kubo, Shinya Takeshima, Mami Nakagawa, Manami Ohta, Saori Kamimura, Eriko Takayama-Watanabe, Akihiko Watanabe, and Kazuo Onitake

Int. J. Dev. Biol. (2010) 54: 591-597

Signalling molecules involved in mouse bladder smooth muscle cellular differentiation

Benchun Liu, Dongxiao Feng, Guiting Lin, Mei Cao, Yuet Wai Kan, Gerald R. Cunha and Laurence S. Baskin

Int. J. Dev. Biol. (2010) 54: 175-180

Nuclear reprogramming in zygotes

Chanchao Lorthongpanich, Davor Solter and Chin Yan Lim

Int. J. Dev. Biol. (2010) 54: 1631-1640

Faithful reprogramming to pluripotency in mammals - what does nuclear transfer teach us?

Julien Maruotti, Alice Jouneau and Jean-Paul Renard

Int. J. Dev. Biol. (2010) 54: 1609-1621

Gonad-stimulating substance-like molecule from the radial nerve of the sea cucumber Hideki Katow, Tomoko Katow and Akihiko Moriyama

Int. J. Dev. Biol. (2009) 53: 483-491

Cyclin B2/cyclin-dependent kinase1 dissociation precedes CDK1 Thr-161 dephosphorylation upon M-phase promoting factor inactivation in Xenopus laevis cell-free extract

Franck Chesnel, Franck Bazile, Aude Pascal and Jacek Z. Kubiak

Int. J. Dev. Biol. (2007) 51: 297-305

Remodeling of sperm chromatin induced in egg extracts of amphibians.

C Katagiri and K Ohsumi

Int. J. Dev. Biol. (1994) 38: 209-216

The influence of mouse sera, regenerating liver extracts and bacterial products on the abilities of different cells in vitro.

N Zarkovic, M Osmak, D Novak, N Lers and M Jurin

Int. J. Dev. Biol. (1991) 35: 239-249

Hormonal factors from the mammalian pineal gland interfere with cell development in Hydra.

W A Müller, C Bartsch, H Bartsch, I Maidonis and E Bayer

Int. J. Dev. Biol. (1998) 42: 821-824
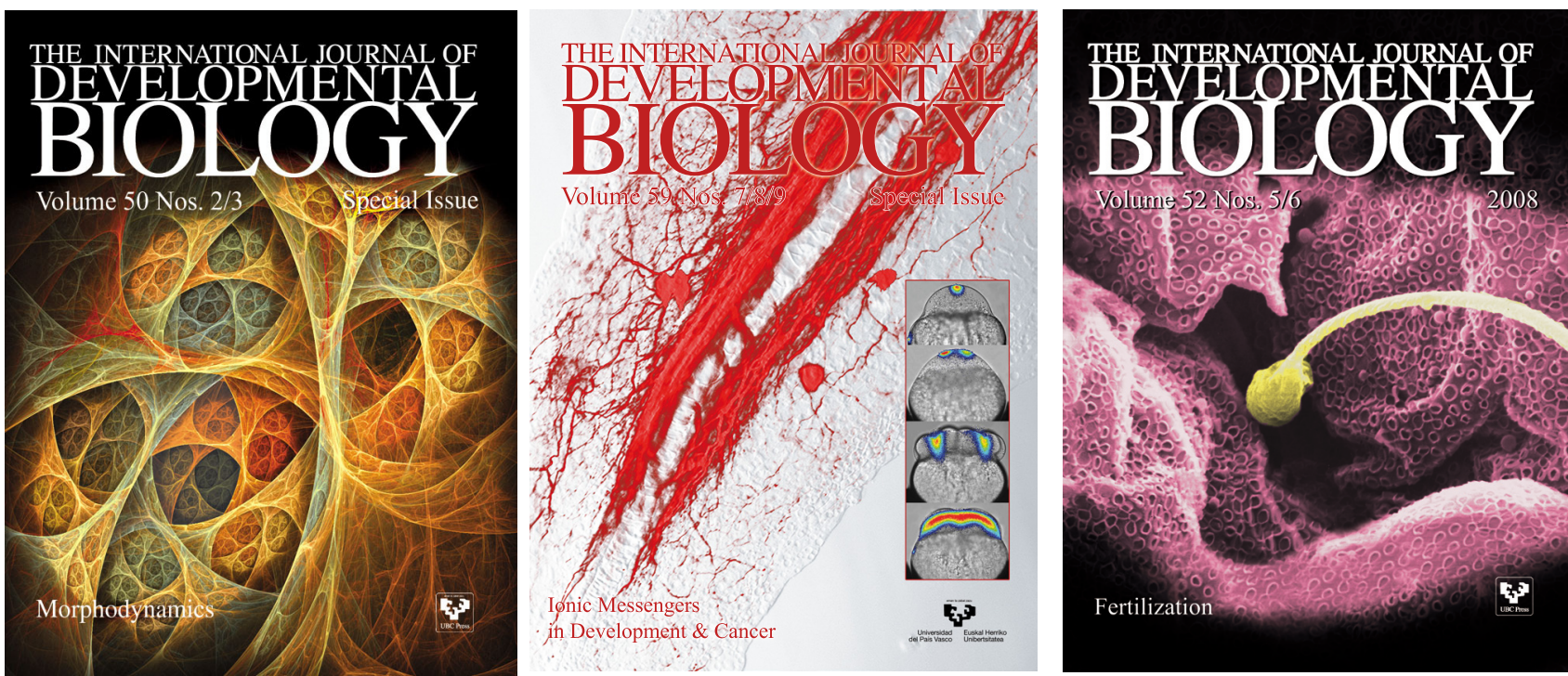